\title{
Computational Tessellation of Freeform, Cut-Stone Vaults
}

\author{
Matthias Rippmann ${ }^{1}$ (D) Philippe Block ${ }^{1}$
}

\begin{abstract}
Contemporary innovations in structural form-finding and fabrication techniques are leading to design of freeform masonry architecture. These new forms create new challenges in laying out tessellation patterns, especially if structural, fabrication and construction requirements as well as aesthetical considerations are taken into account. Addressing these challenges, we review historic stone-cutting strategies and their geometric principles, forming the base for the development of two new discretisation approaches for given thrust surfaces, allowing for various degrees of user control. First, we introduce a tessellation approach based on primal, anisotropic triangular meshes and their dual counterparts. Second, an alternative tessellation approach based on transverse cutting curves is presented. Using a simple set of geometric rules, both methods enable the design of rigid, staggered bonds with locally force-flow aligned block configurations to avoid sliding failures. For this research, the tessellation design of the Armadillo Vault, an unreinforced, dryassembled, cut-stone stone shell, served as a case study to demonstrate the feasibility of our methods in the context of a full-scale architectural project.
\end{abstract}

Keywords Vault design · Freeform · Dry-set masonry · Shell · Vault · Tessellation · Stereotomy $\cdot$ Discretisation $\cdot$ Computational methods

Matthias Rippmann

rippmann@arch.ethz.ch

Philippe Block

block@arch.ethz.ch

1 Block Research Group, Institute of technology in Architecture, ETH Zurich, Stefano-FransciniPlatz 1, HIB E 46, 8093 Zurich, Switzerland 


\section{Introduction}

Historically, stone cutting of masonry architecture involved expertise in structural and geometric analysis of architectural shapes. Due to the negligible tensile capacity of stone, the equilibrium of masonry structures is achieved through geometry, guaranteeing structural form that is in equilibrium predominately through compression forces (Block et al. 2006). Since cut-stone blocks, also known as voussoirs, are of course limited in size, masonry structures depend on the assembly of multiple units to form a stable structural system. Therefore, the precise dressing and arrangement and individual elements is of great importance for the structural integrity of masonry. In this context, stereotomy is deeply tied to aspects of statics, which must be considered for the realisation of stone structures and discrete funicular structures in general. A particular challenge is the design of structurallyinformed segmentations or tessellations of funicular forms. This research presents new discretisation approaches based on a simple set of geometric rules, derived from the analysis of historic techniques, structural requirements and fabrication constraints. Beyond patterns that fit recognisable topologies or primitives, these new approaches allow the flexible design of tessellation patterns for freeform masonry architecture techniques (Fallacara 2006; Clifford and McGee 2013; Rippmann et al. 2012; Schwartz and Mondardini 2014; Rippmann et al. 2016).

This paper is structured as follows: In "Learning from Historic Stone Masonry Techniques", related research on historic stone masonry structures and digital discretisation techniques is reviewed. "Objectives and Approaches to Tesselations" states the objectives for this research. Based on the defined objectives, "Tessellations Based on Triangular Meshes" and "Tessellations Based on Transverse Cutting Curves" presents two new discretisation approaches for given thrust surfaces. In "Case Study: Tessellation of the Armadillo Vault", the feasibility of our methods is verified in the context of a full-scale architectural project. Finally, conclusions are drawn in last section.

\section{Learning from Historic Stone Masonry Techniques}

To ensure the stability of masonry structures, their design has always been based on rules of geometry (Huerta 2001; Aita 2003). These rules have been applied to design the overall form of vaulted structures, but also to determine the respective cutting strategies for voussoirs constituting such discrete assemblies. A particular problem was the determination of the inclination of the joints with respect to the intrados and extrados of a masonry structure. In general, early theories for the design of joint configurations, as illustrated, for example, in Fig. 1, do not represent an ideal structural solution. They only approximate this ideal solution, in which all joint lines are aligned perpendicular to the line of thrust to avoid sliding between voussoirs when considering no friction at interfaces.

Based on Hooke's study of the catenary curve (1676), the developed understanding of the line of thrust within an arch allowed for the study of possible equilibrium solutions. This understanding led to theoretical studies on the ideal 
(a)

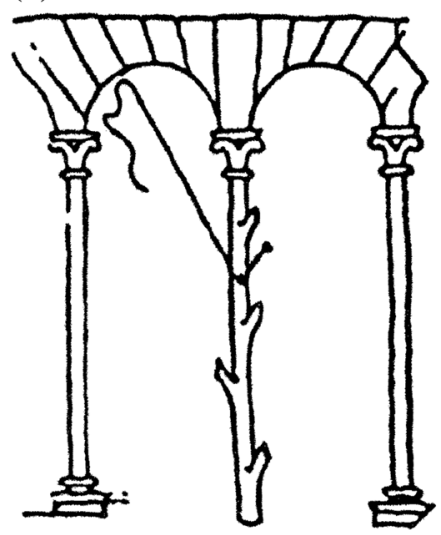

(b)

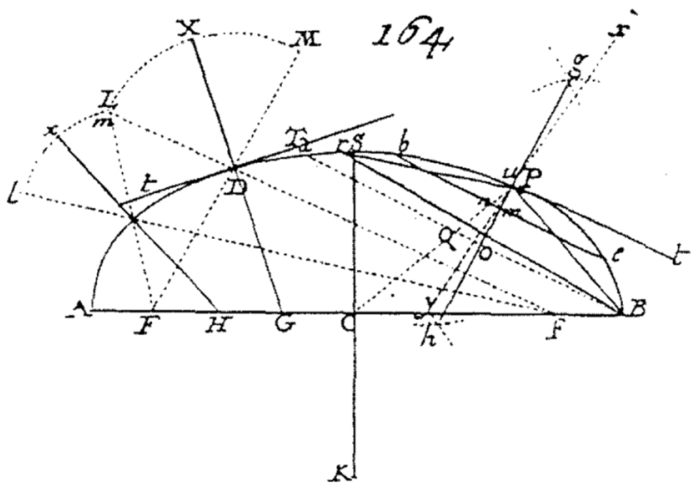

Fig. 1 Tracing voussoir joints using a rope for a a pair of arches with a suspended intermediate capital (Villard de Honnecourt's Carnet, 13th century); and b using drawing techniques to construct joints perpendicular to the intrados of an arch (Milliet de Challes 1674)

shape of arches and domes devoted to the design of the thickness of masonry structures and the ideal orientation of their joint lines (Ageno et al. 2004). By the end of the eighteenth century, such theories on the design and analysis of arches were well developed (Heyman 1972). This understanding helped to align joint lines in section based on structural considerations for simple vaults. However, the structurally-informed alignment of voussoir courses for geometrically more irregular structures was not addressed in such theories. In this context, historic masonry skew arch bridges are an interesting subject of study due to their complex geometry. A skew arch bridge makes it possible to span an obstacle at some angle other than a $90^{\circ}$. This geometry is derived from those of symmetrical arches by distortion in a horizontal plane.

The stability of a skew arch, for which the courses of voussoirs are aligned in the same manner as for a non-distorted arch (Fig. 2a) is reduced with an increasing angle of skew. This configuration is known as a false skew arch and increases the risk of sliding between parallel courses due to the fact that resultant forces between voussoirs of neighbouring courses are not perpendicular to their load-transferring faces. For small arches with a small angle of skew and moderate loading, such configurations proved to be sufficient to guarantee the safety of the structure (Culley 1886). Large spans, a higher angle of skew and heavier loading demand different tessellation strategies. The tessellation of a helicoidal skew arch (Fig. 2b), features courses of voussoirs parallel to one another, following parallel helical curves between the abutments. For arches with constant radius, since the helical courses run parallel to each other, all voussoirs with the same length have the same geometry and can be dressed using only one set of templates. The alignment of the courses of a helicoidal skew arch is only an approximation to the ideal. They are perpendicular to the thrust at the crown of the arch only, but become more and more oblique to the local thrust between voussoirs of neighbouring courses the nearer they are to the abutments. In search of a structurally ideal method, Scottish 

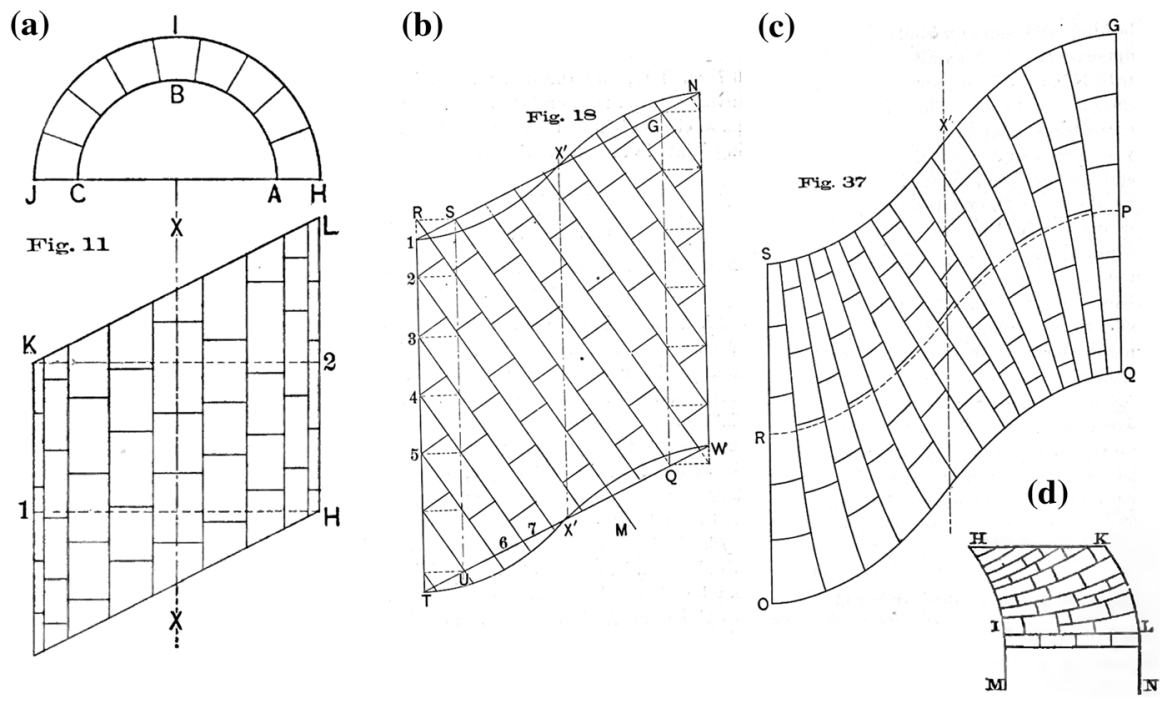

Fig. 2 Types of skew arches: a false skew arch, b helicoidal skew arch, and c logarithmic skew arch (Culley 1886). d Intermediate voussoir courses to avoid very large voussoirs in logarithmic skew arches. Images: (Rankine 1862: 431, Fig. 184)

mathematician Edward Sang (1805-1890) developed the logarithmic method for skew arches (Fig. 2c). In this method, voussoir courses follow the "orthogonal trajectories of curves of pressure" (Rankine 1862) resulting in load-transferring faces perpendicular to the local resultant force between neighbouring voussoirs. In such a configuration the courses are called the equilibrated courses, guaranteeing the static equilibrium of the arch without the need for friction. This approach results in courses that become thinner towards the side of one abutment and thicker towards the other. As a result, it is often necessary to introduce intermediate voussoir courses where the courses become thicker to avoid very large voussoirs, in order to ease fabrication and handling (Fig. 2d) (Rankine 1862).

In his book A Manual of Civil Engineering (1862), Scottish engineer William John Macquorn Rankine (1820-1872) provided a valuable summary of the structural considerations for general stone masonry construction applied in civil engineering at that time. He states that in masonry structures a series of courses should be "perpendicular or as nearly perpendicular as possible to the direction of the pressure which they have to bear; and to avoid all long continuous joints parallel to that pressure..." (Rankine 1862: 52) Certainly for contemporary structural masonry, but also for other discrete assemblies built from alternative material, using, for example, prefabricated concrete elements, Rankine's assumptions are still valid today. However, to generalise such geometric rules for the discretisation of complex funicular shapes a profound understanding of related computational discretisation methods is essential. Such methods will be discussed next. 


\section{Structurally Informed, Computational Discretisation}

This review of computational discretisation methods focuses on algorithms to determine the orientation and arrangement of structural members by optimising mesh topologies and nodal positions to obtain structurally efficient grid configurations. Mostly, such methods, as, for example, presented in Schiftner and Balzer (2010), are designed for the layout of gridshell configurations and thus cannot be directly applied to generate tessellation patterns for discrete funicular shells. Nonetheless, some methods, as, for example, presented in Pietroni et al. (2015), suggest interesting applications of a hexagon-dominant layout for discrete assemblies due to its inherently staggered configuration. Another approach to find optimised staggered bonds has been presented in Bärtschi and Bonwetsch (2013). Addressing brickwork, their approach can be used to find sufficient overlaps of bricks in neighbouring courses with differing lengths. However, it is limited to structures for which all courses are horizontal and consist, with a few exceptions, of standard-sized elements. A combination of requirements to generate tessellations that are locally aligned to the force flow and structurally bonded by a staggered layout is discussed in Panozzo et al. (2013) and Oval et al. (2017). Similar to approaches presented in Schiftner and Balzer (2010), the methods include the generation of a force-aligned quadrilateral meshes based on an assumed force flow for a given shell surface (Fig. 3a), but uses a greedy algorithm that converts the quad mesh into an hexagon-dominant, staggered tessellation by removing "every second edge" (Fig. 3b).

The approach can be used to quickly generate structurally feasible tessellations on complex surfaces. However, due to competing constraints during the process of removing of edges, continuous joints over two or more course are likely to occur, locally weakening the structural bond. Additionally, abrupt direction changes in the tessellation pattern cannot be avoided because of the $90^{\circ}$ orientation constraint on the initial quadrilateral tessellation. Lastly, the control over the width-length ratio of

(a)

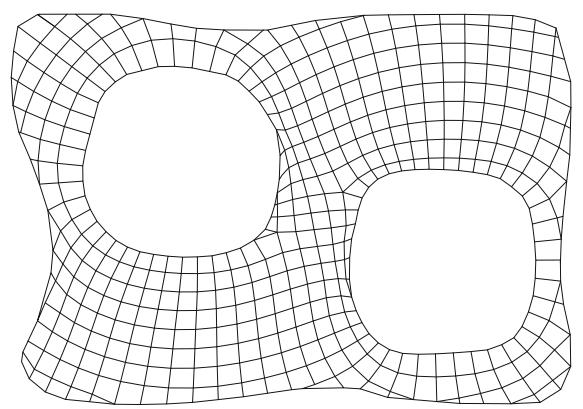

(b)

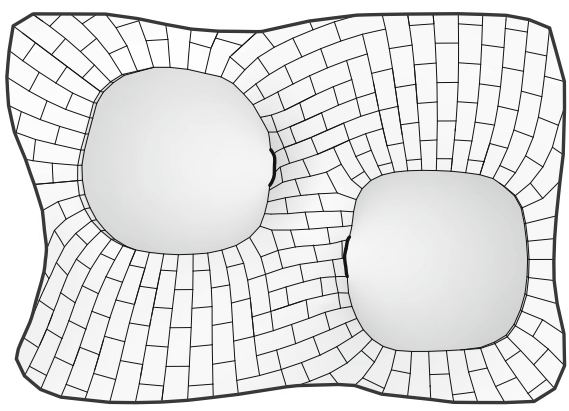

Fig. 3 Tessellation for a discrete masonry model: a a force-aligned mesh consisting of predominantly quadrilateral faces, transformed into b a structurally informed, staggered tessellation by removing "every second edge". Image: Author, after (Panozzo et al. 2013) 
the resulting hexagons, and thus over the dimensions of the discrete elements, is limited.

\section{Objectives and Approaches to Tesselations}

Despite the existence of various historic and computational methods to discretise shell surfaces, none of the approaches reviewed above meets all requirements for flexible, structurally informed tessellations of funicular shells. Most methods discussed focus on fully automated processes, leaving little flexibility for the user to actively control the design process of such tessellations. Therefore, our objective is to develop new computational strategies to explore tessellation designs for cut-stone vaults and discrete funicular structures in general, while taking into account structural requirements and fabrication constraints. Based on the review of historic stone masonry, these requirements were identified and translated into a set of simple geometric rules that can be summarised as follows:

- Voussoirs should be aligned such that the load-transferring contact faces are as perpendicular as possible to the local force flow to prevent sliding failure.

- The tessellation pattern should be staggered or similarly laid out to ensure an interlocking voussoir arrangement.

- The size of voussoirs should be as uniform as possible over the entirety of the surface.

Applying these rules means controlling the layout and spacing of courses as well as the subdivision of each course into a certain number of voussoirs. The basic principle of these geometric operations and their interdependencies is illustrated in Fig. 4 by showing variations of tessellations for a circular and elliptical boundary. Figure 4a shows a basic tessellation of a circular dome by generating equally spaced rings as transverse cutting curves offset to the boundary. They are locally perpendicular to the force flow, defining the courses and hence the height of the voussoirs. A staggered tessellation is generated by dividing each ring into an equal number of parts and then connecting every second division point pair per course. This approach leads to a relatively large variation in the length of the voussoirs. Especially for structures with a larger number of courses, the approach results in infeasible voussoir dimensions towards the centre or support. Figure $4 \mathrm{~b}$ shows a possible solution to avoid the small voussoirs in the two inner courses by connecting only every fourth point pair. The approach results in a structured tessellation but cannot be generally applied to complex shapes. Figure $4 \mathrm{c}$ shows the result of a more general approach, in which each course is individually divided based on a specific voussoir target length while aiming for a sufficient spacing between vertical joints of neighbouring courses and hence guaranteeing a staggered bond. Besides these approaches to keep the length of voussoirs as uniform as possible, additional strategies are necessary to keep the height of voussoirs within a specific range. For example, the elliptical transverse curves in Fig. $4 \mathrm{~d}$ are unequally spaced to guarantee their local, perpendicular alignment to the force flow. This results from competing constraints, i.e., the heights of voussoirs cannot be uniform while 
(a)

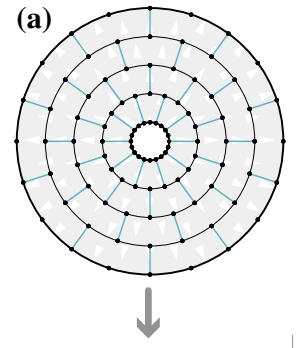

(b)

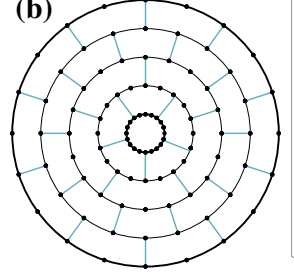

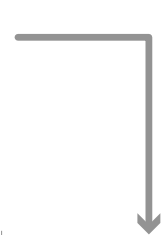

(c)

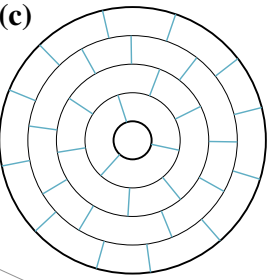

(d)

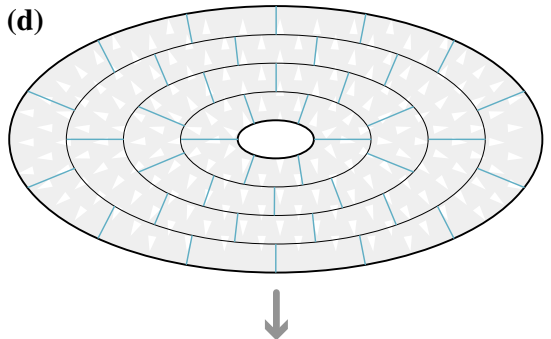

(e)

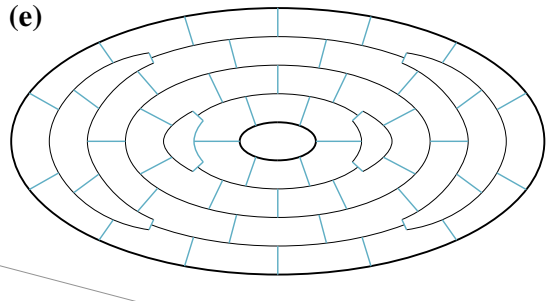

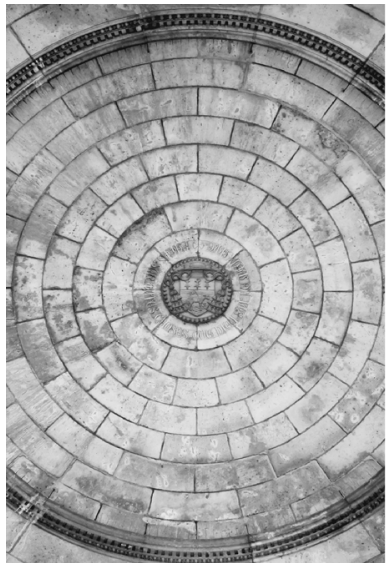
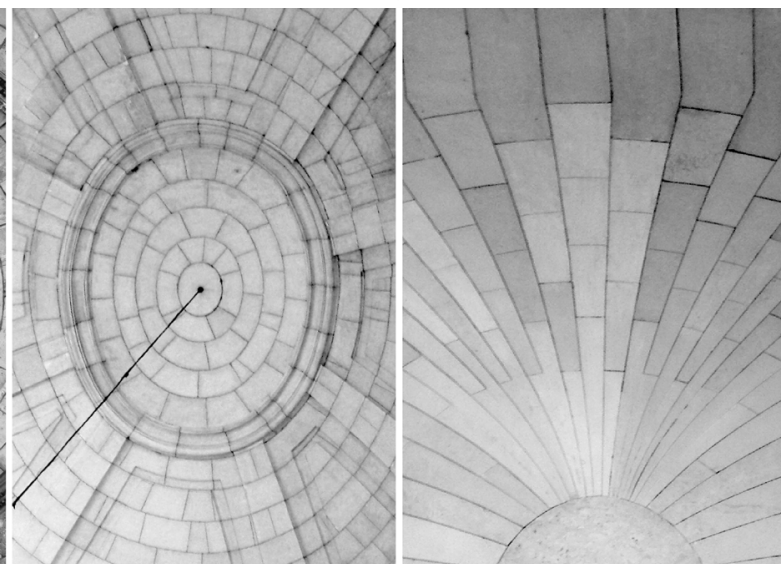

Fig. 4 Study of tessellation geometries facilitating a staggered bond and uniform voussoir dimensions for a a circular and $\mathbf{d}$ elliptical dome through examination of historic structures: $\mathbf{b}$ entrance dome at the Basilica of the Sacré Coeur, Paris, France (1914), c elliptical dome of the Chapelle de l'Oratoire, Avignon, France (1749), and e the grand staircase at the Palais Rohan, Bordeaux, France (1784). Photos and images: authors

enforcing their load-bearing contact faces to be normal to the local force direction. To accommodate the large gaps between the transverse curves along the major axis of the elliptical boundary, additional courses are locally added as shown in Fig. 4e.

Based on this analysis and the rules defined above, two alternative tessellation approaches for discrete funicular shells were developed and implemented as software tools using the programming language Python and the compas framework (Van Mele 2017). Both approaches will be discussed in the following subsections. 


\section{Tessellations Based on Triangular Meshes}

The tessellation design process presented in this section is based on primal, triangular meshes and their dual counterparts. The discretisation approach uses triangular meshes to generate hexagon-dominant tessellations that inherently form a staggered bond. However, besides the staggered configuration, the stability of such hexagonal structures depends on the orientation and alignment of the tessellation based on the local force flow. For example, a closed, funicular dome structure, based on an isotropic, hexagon-dominant tessellation, continuously supported at its circular boundary, is not likely to collapse due to sliding failure (Fig. 5a).

Despite its arbitrarily oriented tessellation, any significant, local sliding of voussoirs is blocked by their neighbours, thanks to their mutually kinematically constraining configuration (Estrin et al. 2011; Tessmann 2013). In contrast, structures based on regular, hexagonal tessellations with openings and open edge arches (Fig. 5b), generally have no self-interlocking configuration. Voussoirs of the barrel vault shown might start sliding in the direction of least resistance, i.e., towards the closest "unsupported" edge arches, which results in a propagating collapse of the structure. In such a case, the friction angle between the local force vector and load transferring faces determines whether sliding occurs. The friction angle is defined as

$$
\tan \theta=\mu_{s},
$$

where $\theta$ is the angle between the normal of the load-transferring face and the local force vector and $\mu_{s}$ is the static coefficient of friction between the elements. For example, $\mu_{s}$ for masonry is $0.6-0.7$, which results in a friction angle of approximately $31^{\circ}-35^{\circ}$. Consequently, hexagonal tessellation geometries need to be modified based on the force flow in the structure such that the angle between the normal of a load-transferring face and the local force vector is well below the given friction angle. The vault in Fig. 5c shows that an anisotropic, hexagonal tessellation, stretched in the direction perpendicular to the force flow, minimises this angle and thus the risk of sliding failure.

Extending the work of Botsch and Kobbelt (2004) on the isotropic remeshing of triangulated meshes, an anisotropic triangulation method was developed and implemented in a design framework for anisotropic, hexagon-dominant tessellation geometries. The fundamental idea of the presented approach is to locally control the

(a)

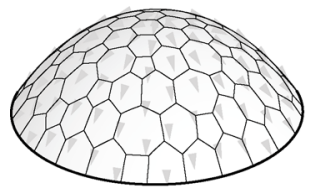

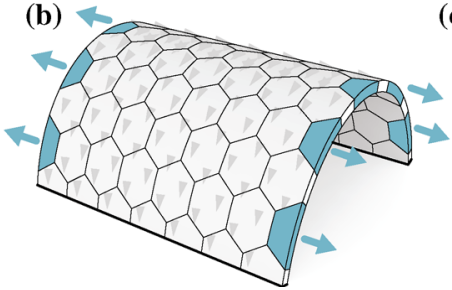

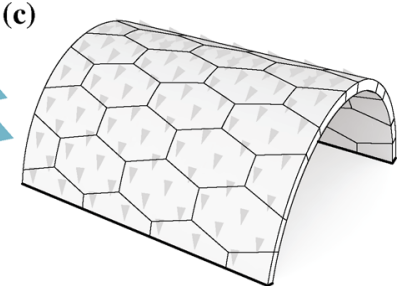

Fig. 5 Isotropic, hexagonal-dominant tessellation geometries for a a dome and b a barrel vault. c Anisotropic, hexagonal-dominant tessellation geometry for a barrel vault. Images: authors 
distortion of a triangulated mesh based on the local force flow such that its dual mesh results in a structurally informed and fabrication-aware tessellation geometry. The flow diagram in Fig. 6 provides a visual overview of the successive steps of the approach, briefly introduced as follows:

(a) A NURBS surface as initial input geometry represents the funicular shape. A sufficiently dense force vector field represents the flow of forces.

(b) The user defines supported and unsupported boundaries.

(c) The supported boundaries are divided based on a user-defined voussoir target length, and the unsupported boundaries are divided based on a user-defined voussoir target height.

(d) Based on these division points on the defined boundaries, an initial mesh is generated using Delaunay triangulation.

(e) Before the mesh is refined, target valencies are assigned to all boundary vertices.

(f) The initially generated mesh is refined based on the input surface, the force vector field, the given target valencies and a user-defined height and length for the voussoirs.

(g) Based on the refined mesh, a hexagon-dominant dual mesh is computed by connecting centroids of neighbouring triangles.

(h) Post-processing procedures are applied to complete the dual mesh by adding special faces along the boundaries. Mesh-smoothing techniques can optionally be used to improve the size uniformity of faces in the resulting dual mesh.

The vector field representing the force flow can be obtained by analysing the local principle strain of an input surface assuming the structural behaviour of a continuous shell (Schiftner and Balzer 2010), directly from a Thrust Network

(a)
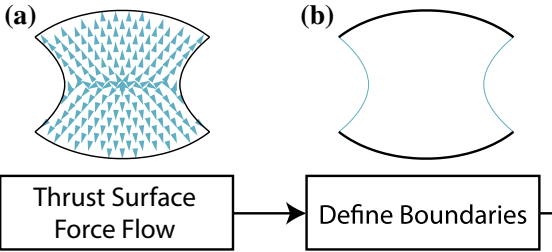

(e)

(e)

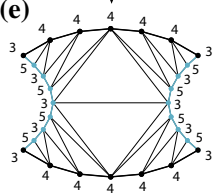

Set Valencies (b)

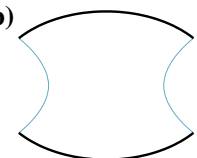

(f)

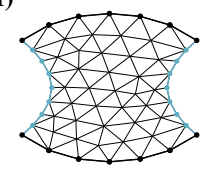

(c)
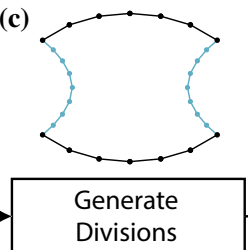

(g)

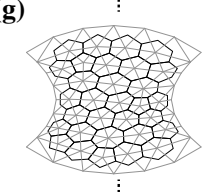

(h)

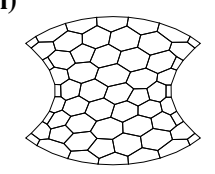

Post-Processing (d)

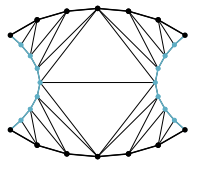

Generate Initial Mesh

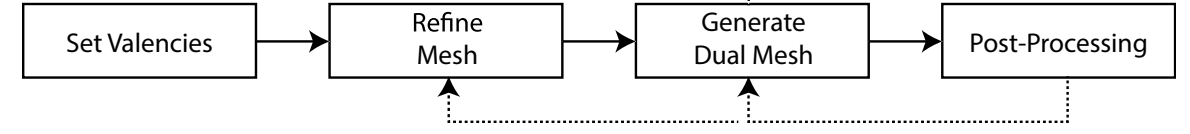

Fig. 6 Flow diagram showing the sequential steps of the developed tessellation algorithm based on anisotropic, triangular meshes 
Analysis (TNA) form-finding process (Block et al. 2014) or from using a geometric approach based on structural heuristics (Panozzo et al. 2013). The other operations in steps (a)-(d) of the tessellation design process are straightforward and will not be discussed in this paper.

The target valencies defined for the initial mesh in Fig. 6e play an important role for its refinement. This refinement process, which will be discussed in detail below, optimises the topology of the initial mesh by minimising its valency error. The valency error is the absolute difference between the target valencies and the actual valencies for all vertices. For example, the target valencies for an isotropic, triangular mesh is 6 for internal vertices and 2-5 for boundary vertices depending on their inner corner angle (Tam and Armstrong 1991). The initial Delaunay mesh in Fig. 7a results from the division points along the unsupported boundaries (left/right) and the fixed (supported) boundaries (top/bottom). Based on the general definition of vertex valencies, the initial mesh and the unidirectional force vector field, a refined anisotropic mesh is computed (Fig. 7b). The resulting triangulation and its dual, hexagon-dominant mesh are stretched perpendicular to the force vector field. However, the alignment and staggered configuration of the tessellation (dual mesh) are not ideal due to the poor interlocking of the elements along unsupported edges. To avoid this, we use an alternative set of target valencies, as shown in Fig. 7c. The vertices on support boundaries and boundary corners are defined as before, whereas the valencies of vertices along unsupported boundaries are alternatingly assigned to three and five. This specific valency assignment results in more suitable tessellation geometries with better interlocking properties along the unsupported boundaries. Note that the assigned target valencies can differ from the actual valencies of the refined and optimised mesh.

After the initial mesh has been computed and the valencies for the boundary vertices set, the mesh refinement process starts. The remeshing algorithm used for this research extends the work of Botsch and Kobbelt (2004) to facilitate the generation of anisotropic meshes based on a local force vector field. The iterative algorithm uses three simple mesh operations commonly used in triangular mesh optimisation: collapsing an edge to a point, splitting an edge by inserting a new vertex and two adjacent edges, and swapping an existing edge (Hoppe et al. 1993).

(a)

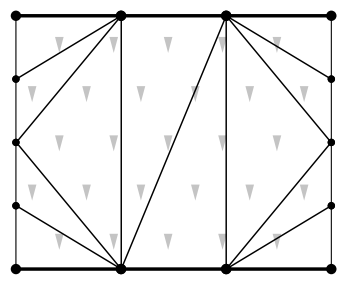

(b)

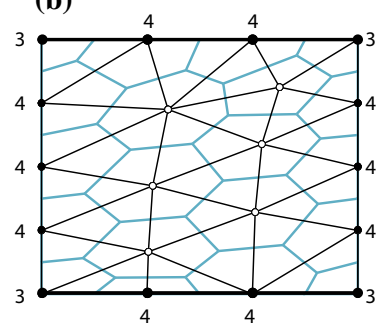

(c)

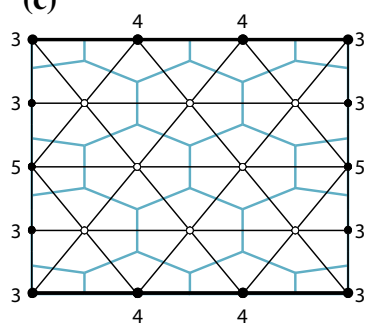

Fig. 7 a The initial mesh in refined using the developed anisotropic meshing approach based on b standard and corner target valencies (Tam and Armstrong 1991) and c alternating target valencies $(3,5)$ for unsupported boundaries, as defined in this research. Images: authors 
Using the thrust surface, the force vector field, the initial coarse mesh, the target voussoir width $w$ and the target voussoir height $h$, the following steps are performed:

1. Split all mesh edges that are longer than $4 / 3 l_{t}$ at their midpoint. As described later, $l_{t}$ is the determined, local target length based on $w, h$ and the angle between the edge and the interpolated, local force vector.

2. Collapse all edges shorter than $4 / 5 l_{t}$ to their midpoint.

3. Flip edges in order to minimize the deviation from valency 6 or the predefined valency for boundary vertices.

4. Relocate vertices on the surface by directional smoothing based on $l_{t}$.

These steps are repeated until the mesh topology no longer changes for several iterations or until a defined number of iterations is reached.

Whether an edge is split or collapsed throughout the iteration steps depends on the difference between its local target length $l_{t}$ and its actual length $l$. Note that $l_{t}$ ranges between the target voussoir width $w$ (Fig. 8b) and the target voussoir height $h$ (Fig. 8e) based on the angle between the corresponding edge and the local, interpolated force vector at its midpoint. The simple examples in Fig. 8 illustrate how the length of a particular edge changes when altering the global direction of the force field. Figure 8 a shows a regular mesh containing six equilateral triangles and its central dual polygon, representing the hexagonal voussoir outline. This configuration is isotropic and all edges have the same length. The meshes in Figs. $8 \mathrm{~b}-\mathrm{e}$ are stretched perpendicular to the unidirectional vector fields using anisotropic scaling.

The two mutually perpendicular scaling axes are aligned with the force vectors. For such transformations, the independent scaling factors $x$ and $y$ for a particular edge are

$$
\begin{aligned}
& x=m\left(\frac{1}{2}(-w-h)+w\right)+\frac{w+h}{2}, \text { and } \\
& y=m\left(\frac{1}{2}(-w-h)+h\right)+\frac{w+h}{2}, \text { and }
\end{aligned}
$$

where $m$ is the interpolated force magnitude in the force vector field based on the midpoint of the edge. This normalised magnitude, ranging between 0 and 1 , is not necessarily proportional to the actual forces in the structure, but can be actively

(a)

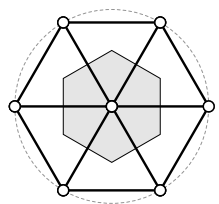

(b)

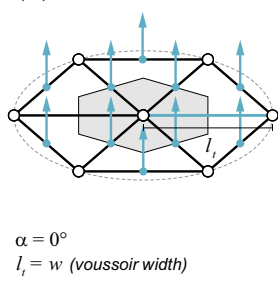

(c)

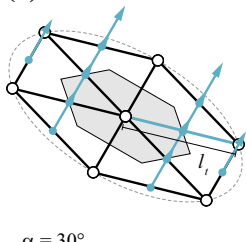

$\alpha=30^{\circ}$ (d)

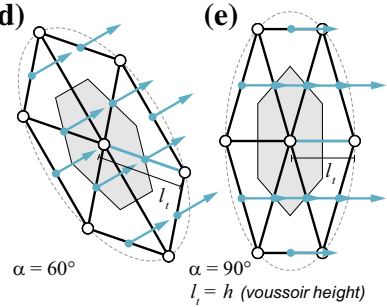

Fig. 8 Based on a an isotropic mesh, b-e anisotropic meshes are obtained, using direction-dependent transformations, informed by the local force flow (arrows) 
defined to locally control the intensity of the anisotropic scaling. This makes it possible to have uniform, non-stretched faces (Fig. 8a) in areas where no directional constraint can be applied, for example, at and close to singularities (Schiftner and Balzer 2010). For the presented method, the two scaling factors $x$ and $y$ are identical for regions where $m=0$. With $x$ and $y$ defined, the local target length for an edge is:

$$
l_{t}=\sqrt{\frac{x^{2} y^{2}}{x^{2}(\hat{\mathbf{e}} \cdot \hat{\mathbf{f}})^{2}+y^{2}\left(1-(\hat{\mathbf{e}} \cdot \hat{\mathbf{f}})^{2}\right)}}
$$

where $\hat{\mathbf{e}}$ is the normalised direction vector of the edge and $\hat{\mathbf{f}}$ is the normalised force vector at its midpoint based on the local force flow. Note that $l_{t}$ is the radius of an ellipse with the semi-major $(x)$ and semi-minor axis $(y)$ at a specific angle based on $\hat{\mathbf{e}}$ and $\hat{\mathbf{f}}$ (Fig. 8). These target lengths are only computed for non-boundary edges and determine if an internal edge needs to be split or collapsed. For edges adjacent to exactly one vertex with a defined valency of 3 on an unsupported boundary, a special target length $1 / 2 l_{t}$ is applied to meet the geometric requirements of the staggered topology along open edge arches (Fig. 7c). Whether an edge is swapped depends on the valencies of the vertices of both its adjacent triangles (swapping is not allowed on boundary edges). An edge is swapped if its updated orientation would result in a lower valency error for these four vertices.

To allow this mesh refinement process to converge and avoid oscillation effects, the vertices need to be redistributed in each iteration. This relaxation process is performed on the input surface and based on the computed edge target lengths using directional smoothing.

Figure 9 shows this tessellation method applied to two irregular thrust surfaces to structurally improve their tessellation patterns according to the presented geometric rules. All interior edges with a deviation angle $<30^{\circ}$ (based on $\mu_{s} \approx 0.6$ for masonry) with respect to the ideal local orientation (perpendicular to the local force flow) are highlighted. Comparing these results with isotropic, non-directed tessellations for the same vaulted shape shows that the total edge length of edges below the defined deviation angles can be increases by $\sim 65 \%$ when using the presented anisotropic remeshing method, i.e., the risk of sliding failure can be reduced significantly. The method requires little manual modelling and makes it relatively easy for less experienced designers to generate structurally informed tessellation geometries.

\section{Tessellations Based on Transverse Cutting Curves}

Applying the method described in "Tessellations Based on Triangular Meshes" on various thrust surfaces revealed that a more controlled tessellation design process is often desirable and led to the development of an alternative tessellation approach. This user-driven procedural method allows controlling the layout of courses and thus the arrangement of voussoirs. The flow diagram in Fig. 10 provides a visual overview of the steps of the method, which can be summarised as follows: 
(a)
anisotropic
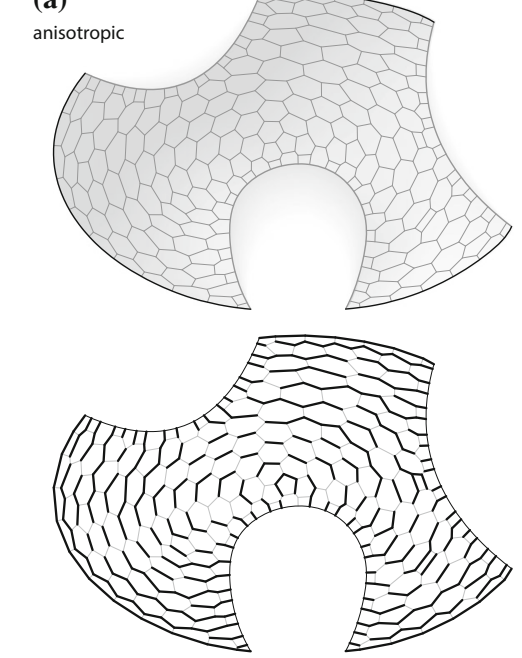

isotropic

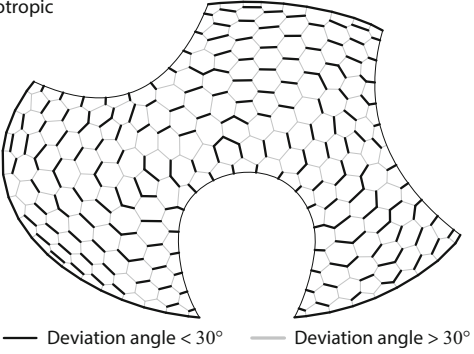

(b) anisotropic
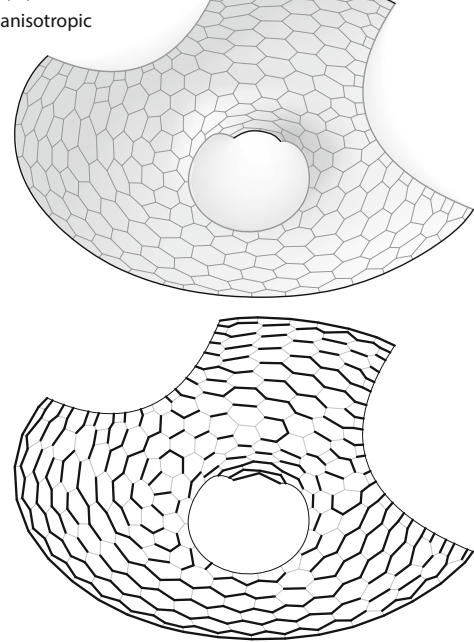

isotropic

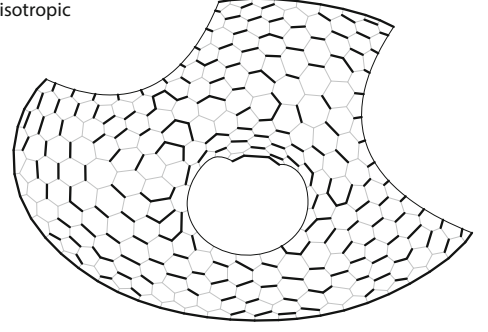

Fig. 9 Visual comparison of anisotropic and isotropic tessellation meshes for two (a, b) irregular thrust surfaces. The edges with deviation angles $<30^{\circ}$ with respect to the ideal local orientation are highlighted. Images: authors

(a) The initial input geometry is the funicular shape represented as NURBS surface. A sufficiently dense force vector field represents the force flow.

(b) Based on the force vector field, the user defines triangular or rectangular regions on the thrust surface. These regions serve as controllable patches to facilitate the subsequent discretisation of complex surfaces with multiple singularities (e.g., at global and local minima and maxima, and saddle points).

(c) Based on a user-defined voussoir target height, the patch boundaries aligned parallel to the local force flow are divided, determining local start and end points to generate the transverse cutting curves (step d) of the respective patches.

(d) The transverse cutting curves are generated on the surface based on the defined division points per patch and the force vector field. The transverse curves 
(a)

(b)

(c)

(d)
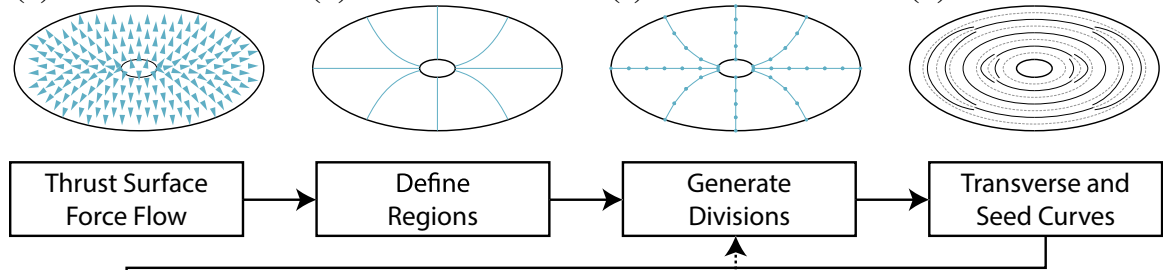

(e)

(f) (g)

(h)
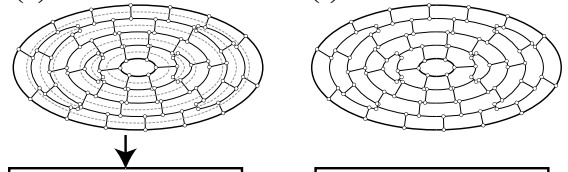

(g)

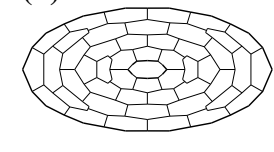

Generate

4

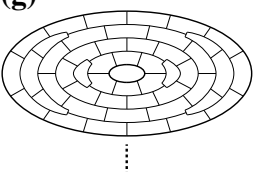

\section{Initial Topology}
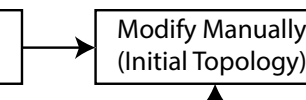
(Initial Topology)

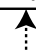

Apply

Post-Processing

Fig. 10 Flow diagram showing the sequential steps of the developed tessellation approach based on transverse cutting curves

define voussoir joints orientated as perpendicular as possible to the local force flow.

(e) Each seed curve lying in between two neighbouring transverse curves is divided based on a specified voussoir length to define the location of equally spaced joint lines. These lines are aligned parallel to the local force flow and define joints between voussoirs in the same course. All joint lines are used to establish an initial starting topology representing the tessellation.

(f) The starting topology can be modified manually.

(g) A balanced staggering is enforced through an automated procedure that maximises the distance between joints of neighbouring courses. The resulting tessellation geometry can be visually and numerically checked by the designer and optionally refined by simply going back to step (f). For changes concerning the dimension of the voussoir, the tessellation process needs to be repeated starting from step (c) or (e).

(h) Post-processing routines can be applied to modify the geometry of faces such that they, for example, have convex boundaries to facilitate certain fabrication techniques.

The initial vector field on the given thrust surface is obtained as previously discussed in "Tessellations Based on Triangular Meshes". The regions defining the course layout are based on structurally-informed block decomposition techniques (Oval et al. 2017) or simply drawn manually by the user.

The layout of the transverse cutting curves based on the boundary curves between neighbouring regions is shown in Fig. 11.

Figure 11 shows two surface patches containing three patch curves parallel $(a, b$, c) and four curves perpendicular (d, e, f, g) to the local force flow. All curves 

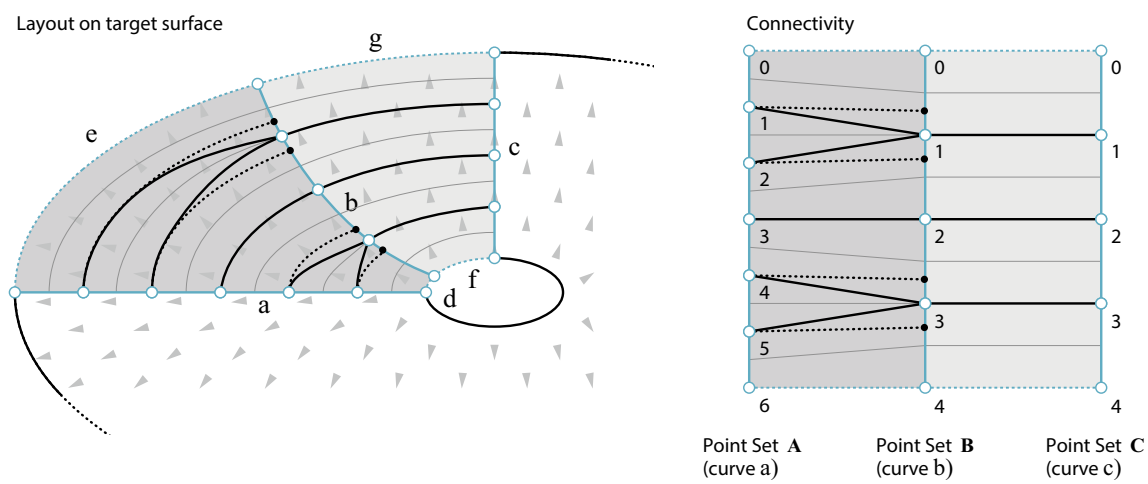

Fig. 11 Transverse cutting curves on one quarter of an elliptical dome (partly shown), based on patch curves (a-e) with different divisions for two patches and their connectivity shown in a graph. Images: authors

parallel to the force flow are divided based on their individual geodesic length and a defined voussoir target height, resulting in three sets of division points $\mathbf{A}, \mathbf{B}, \mathbf{C}$. Due to the elliptical shape, the division number is different for curves a and $b$, making it necessary to "fork" transverse cutting curves. Figure $11 \mathrm{~b}$ shows a simplified connectivity graph, illustrating the transition between two patch curves with different numbers of divisions points. The connectivity is computed using a shortest-path algorithm. The interpolated seed curves (grey), which are used in a later step to generate equally spaced joint lines for a staggered voussoir bond, and the transverse curves (black) are alternately generated, requiring the doubling of the number of divisions per patch curve. Based on the computed connectivity, transverse curves are generated per patch using an optimisation method (Rippmann 2016) to ensure their local alignment perpendicular to the vector field. The dotted transverse curves in Fig. 11 represent alternative curves, which can be generated by simply moving their input points along the corresponding patch curves. The distance and direction of the respective point displacement can be parametrically defined by the user in order to control the resulting transverse curves, resulting in the shape of the corresponding voussoir courses (Fig. 12). The resulting tessellation variations have different consequences regarding fabrication and can be freely adjusted to match the requirements of a particular project.

To form a stable, three-dimensional structural surface, an interlocking, staggered voussoir arrangement is necessary. This is achieved by avoiding alignment of side joints of voussoirs in any course to side joints in the course below. Ideally, a

(a)

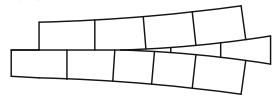

(b)

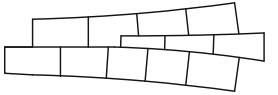

(c)

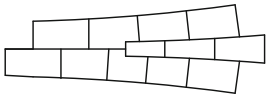

(d)

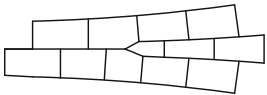

Fig. 12 Parametric changes in the generation of transverse cutting curves result in a-c alternative tessellation geometries. d Optional post-processing can be used to simplify the pattern, or to optimise the voussoirs for fabrication. Images: authors 
voussoir should overlap its neighbours by half of its length. However, this ideal configuration is not generally possible for tessellation geometries applied to doubly curved surfaces, while simultaneously guaranteeing voussoirs of equal lengths, as discussed previously (see Fig. 4). An automated approach was developed to maximise the distance between joints of neighbouring courses, while minimising the difference in length among all voussoirs. For this approach, an initial topology of the tessellation is generated based on the defined voussoir target length to generate equally spaced vertical joint edges. Special alternating division start points are assigned for neighbouring courses perpendicular to openings to guarantee an initial staggered topology along all unsupported edge arches. All parallel joint edges together with the transverse curves form a tessellation mesh topology that is hexagon-dominant to facilitate a staggered pattern. However, the joint edges are poorly distributed, which results in insufficiently overlapping voussoirs, demanding an additional procedure to optimise the staggered bond. The steps of this iterative procedure are shown in Fig. 13 and briefly introduced here:

(a) The initial configuration and starting topology for a tessellation mesh is generated using the previously discussed method. The vertices $\left\{\boldsymbol{v}_{1}, \ldots, \mathbf{v}_{n}\right\}$ are constrained to their corresponding transverse curves, which function as guide curves. The three, exemplary vertices $\mathbf{v}_{i}, \mathbf{v}_{j}$ and $\mathbf{v}_{k}$ are used throughout the subsequent steps and highlighted in Fig. 13.

(b) The location of all non-boundary vertices is optimised such that the spacing of joint edges of neighbouring courses is maximised. For example, the position of vertex $\mathbf{v}_{j}$ is updated to the projected centroid of its adjacent vertices $\mathbf{v}_{i}$ and $\mathbf{v}_{k}$ on the same guide curve.

(c) The lengths of edges connecting vertices on the same guide curve are bound to a minimum of a third of the voussoir target length and to a maximum of half of the voussoir target length. For example, after performing step (b), the distance between $\mathbf{v}_{i}$ and $\mathbf{v}_{j}$ falls below the defined minimum. Consequently, both

(a)

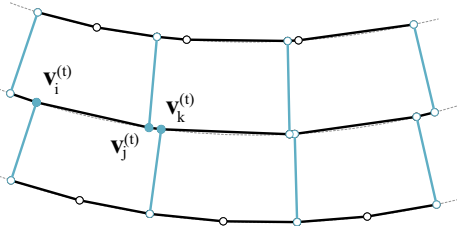

(c)

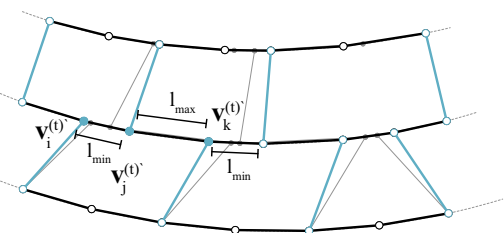

(b)

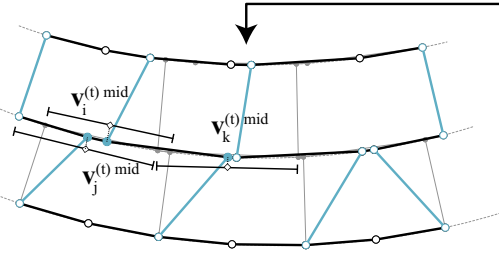

(d)

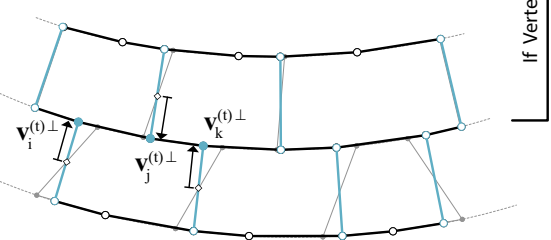

Fig. 13 a-d The steps of one iteration of an automated procedure to redistribute a given tessellation topology, aiming for an aligned, staggered tessellation geometry with sufficiently overlapping voussoirs. Images: authors 
vertices are spaced further apart on the guide curve, aiming for a distance that exceeds the allowed minimum. This process is performed simultaneously for all edges exceeding or falling below the defined length bounds.

(d) Joint edges connecting two neighbouring transverse curves shall be parallel to the local force flow, i.e., corresponding edges must be as perpendicular as possible to both transverse curves. This is guaranteed by projecting their midpoints on both transverse curves. Subsequently, the coordinates of the end vertices of these edges are updated based on the projected midpoints.

Steps (b) to (d) are repeated, using a time step $t$, until the magnitudes of all residuals $\forall(i) \in\{1, \ldots, n\}, \mathbf{v}_{i}^{t}-\mathbf{v}_{i}^{t-1}=\mathbf{r}_{i}$ fall below a defined threshold value.

Using this method, the voussoirs of neighbouring courses overlap by at least a third of their lengths and their overall dimensions remain relatively uniform. In the example shown in Fig. 13, the number of vertices of one face deviates from the ideal of six vertices per face, i.e., the voussoir overlaps not just two but three neighbouring voussoirs. These required irregularities in the pattern explain why the lengths of edges connecting vertices of the same transverse curve are bound to a minimum of a third of the voussoir target length.

The method has been tested through numerous tessellation designs for various thrust surfaces. Four test shapes with corresponding discretisation layouts are shown in Fig. 14. The resulting patterns are smoothly distributed and comply with the defined structural requirements. Figure 15 shows the exploration of a series of

(a)

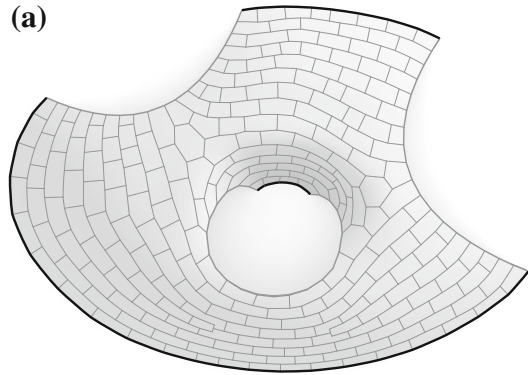

(c)

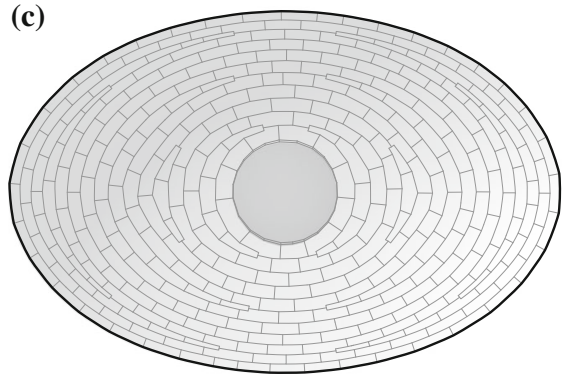

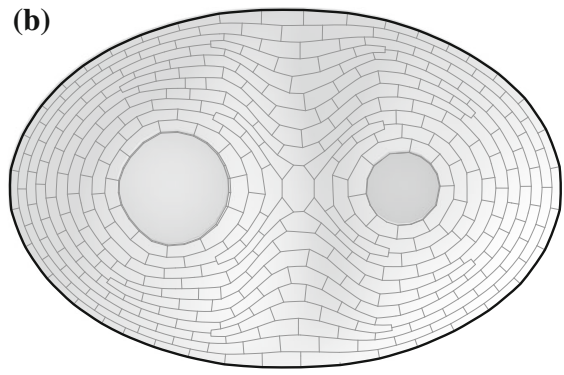

(d)

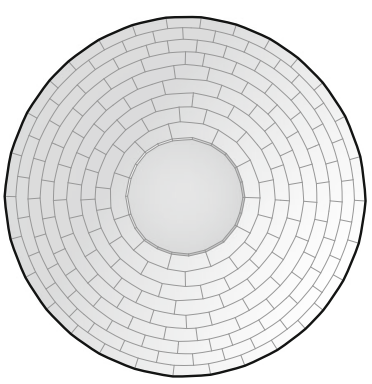

Fig. 14 a-d Resulting tessellation patterns for four freeform thrust surfaces with varying boundary and support conditions 

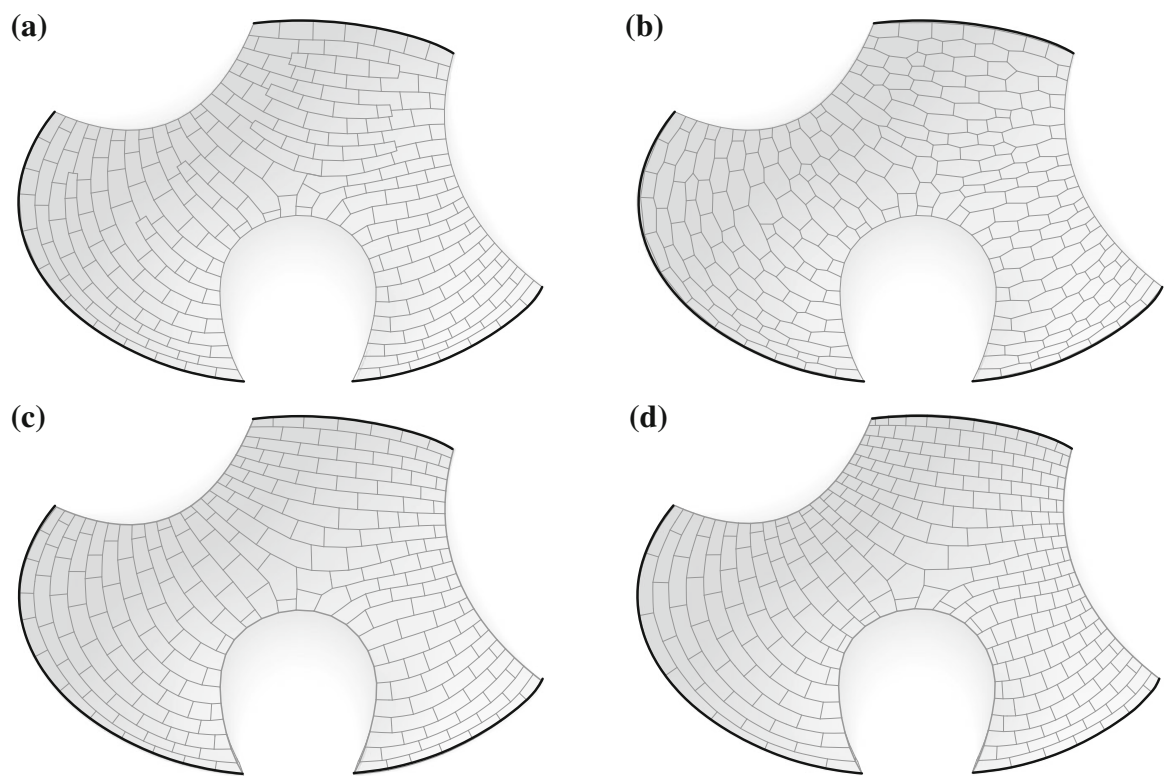

Fig. 15 a-d Exploring different tessellation patterns for the same thrust surface by changing various input parameters. Images: authors

patterns, demonstrating the flexible control of the tessellation design by changing various input parameters.

\section{Case Study: Tessellation of the Armadillo Vault}

The Armadillo Vault was an unreinforced, dry-assembled, cut-stone stone shell constructed by the Escobedo Group in the Corderie dell'Arsenale for the 2016 Architecture Biennale (Fig. 16). The doubly curved vault consisted of 399 individual limestone voussoirs assembled without mortar or other structural connections (Rippmann et al. 2016). The vault stands in compression and spans a total area of $75 \mathrm{~m}^{2}$ with three supports along its boundary and one support in the middle.

The voussoir geometry results from a tessellation geometry designed using the methods presented in this paper. One of the most challenging aspects of the design of the structure was to generate a structurally informed tessellation pattern that eliminates the risk of sliding failure. Additionally, the design of the tessellation pattern must take into account the following fabrication constraints. First, the maximum allowed weight of the voussoirs was limited to $45 \mathrm{~kg}$ on the top and $135 \mathrm{~kg}$ close to the supports, resulting in face size constraints for the final tessellation mesh. Second, the use of circular blades demanded a convex cutting geometry along the interfaces, and thus convex mesh faces, to avoid selfintersections with the blade trajectory. 


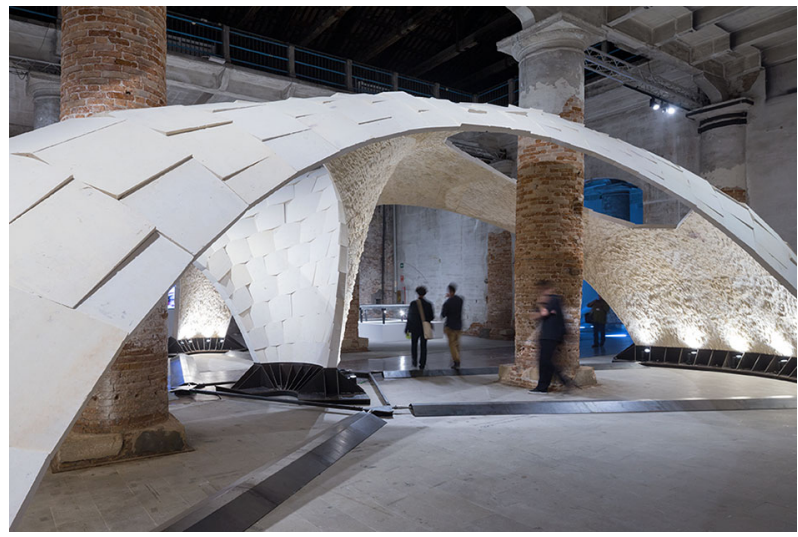

Fig. 16 The Armadillo Vault in the Corderie dell'Arsenale of the 2016 Architecture Biennale in Venice. Photo: Iwan Baan, used with permission
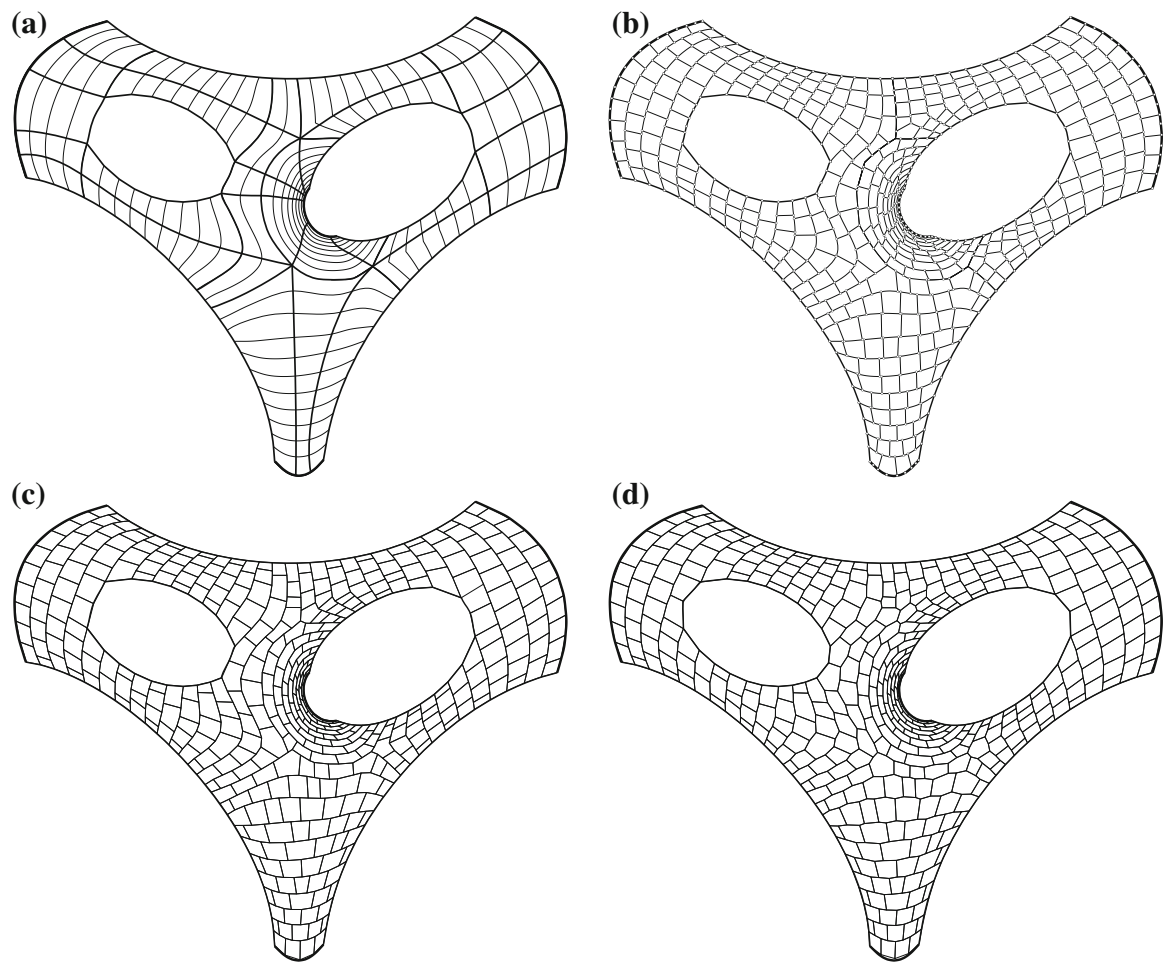

Fig. 17 Overview of the tessellation design: a the quadrilateral patches and course lines on the thrust surface, $\mathbf{b}$ the initial staggered topology, $\mathbf{c}$ the optimised tessellation bond, and $\mathbf{d}$ the final tessellation of the thrust surface with convex mesh faces. Images: authors 
Due to the number of constraints and the need to be able to carefully control the appearance of the prominently exposed tessellation pattern, we decided to use the tessellation approach based on transverse cutting curves discussed in "Tessellations based on transverse cutting curves". Figure 17 shows the steps performed during the process of designing the tessellation geometry starting from the structural surface of the vault. With the help of block decomposition techniques, 32 patches were defined, forming a coarse quad mesh with three internal singularities based on the force vector field and support conditions (Fig. 17a). Within these patches, the course lines were generated based on a variable voussoir target height of $80 \mathrm{~cm}$ at the supports and $40 \mathrm{~cm}$ at the top. Subsequently, an initial staggered topology was generated based on a voussoir target width of $50 \mathrm{~cm}$ (Fig. 17b). Subsequently, we improved the geometry of the mesh using our iterative optimisation method to obtain a nicely balanced tessellation bond with sufficient overlaps between voussoirs in neighbouring courses (Fig. 17c). With small manual adjustments on the initial topology, this optimisation step was repeated until a satisfactory result was found. Finally, post-processing operations were applied to enforce the convexity of all mesh faces (Fig. 17d). This was achieved by scaling the joint lines aligned parallel to the force flow based on a user-defined scale factor and proportional to the course height. As a result, the degree of convexity was increased towards the top, forming smoother transitions around the singularities.

\section{Conclusions}

We have reviewed and analysed historic stone-cutting strategies in order to define a set of geometric rules to be considered in the design of tessellations for freeform masonry vaults. This research has shown that there is no single, most optimal tessellation design for such shapes, since, in addition to technical constraints, more subjective architectural requirements need to be considered as well. Hence, two alternative approaches were presented to account for the various degrees of user control and interaction. First, we introduced a tessellation approach based on primal, anisotropic triangular meshes and their dual counterparts, which require only limited manual modelling due to the increased level of automation. Second, a procedural tessellation approach based on transverse cutting curves was presented, which can be controlled carefully through individual, computer-assisted, stepwise optimisation. Finally, we verified the feasibility of our approach by designing the tessellation geometry for a full-scale, freeform masonry vault.

\section{References}

Aita, Danila. 2003. Between geometry and mechanics : A re-examination of the principles of stereotomy from a statical point of view. Pp. 162-170 in: Proceedings of the First Conference of the Construction History Society. Madrid. 
Ageno, Alessio, Andrea Bernabò, Federico Foce, and Anna Sinopoli. 2004. Theory and history of the thrust line for masonry arches. A brief account. Pp. 1-10 in: 4th. International Conference on Arch Bridges, P. Roca and E. Oñate, eds. Barcelona.

Bärtschi, Ralph and Bonwetsch, Tobias. 2013. A Stretcher Bond with Defects Applied to a Hyperboloid. Pp. 37-42 in: L. Hesselgren et al. eds. Advances in Architectural Geometry 2012. Vienna: Springer Vienna.

Block, Philippe. Matt Dejong, and John Ochsendorf. 2006. As Hangs the Flexible Line: Equilibrium of Masonry Arches. Nexus Network Journal 8(2): 13-24.

Botsch, M. and Kobbelt, L. 2004. A remeshing approach to multiresolution modeling. P. 185 in: Proceedings of the 2004 Eurographics/ACM SIGGRAPH symposium on Geometry processing SGP'04.

Clifford, Brandon and Wes McGee. 2013. La Voûte de LeFevre. Pp. 122-127 in: L. C. Werner, ed., [En]Coding Architecture: The Book. Carnegie Mellon University School of Architecture.

Culley, J. L. 1886. Theory of the Construction of Helicoidal Oblique Arches. New York: D. Van Nostrand.

Estrin, Yuri, Arcady Dyskin, and Elena Pasternak. 2011. Topological interlocking as a material design concept. Materials Science and Engineering: C (April 2011):1-55.

Fallacara, Giuseppe. 2006. Digital stereotomy and topological transformations: reasoning about shape building. Pp. 1075-1092 in: M. Dunkeld et al. eds., Proceedings of the Second International Congress on Construction History..

Heyman, Jacques. 1972. Coulomb's Memoir on Statics: An Essay in the History of Civil Engineering, Cambridge University Press.

Hooke, Robert 1676. A Description of Helioscopes, and Some Other Instruments. London: Printed by T.R. for John Martyn.

Hoppe, Hugues, Tony DeRose, Tom Duchamp, John McDonald, and Werner Stuetzle. 1993. Mesh optimization. Pp. 19-26 in: SIGGRAPH'93 Proceedings of the 20th annual conference on Computer graphics and interactive techniques. New York: ACM New York.

Huerta, Santiago. 2001. Mechanics of masonry vaults: The equilibrium approach. Pp. 47-70 in: $3 r d$ International Seminar in Historical Constructions, Guimarães, Portugal. Guimarães.

Milliet de Challes, Claude François. 1674. Cursus seu mundus mathematicus, 3 vols. Lyon.

Oval, Robin, Matthias Rippmann, Tom Van Mele, Olivier Baverel, Philippe Block. 2017. Patterns for Masonry Vault Design. In Proceedings of the IASS Symposium 2017. Hamburg.

Panozzo, Daniele, Philippe Block, and Olga Sorkine-Hornung. 2013. Designing Unreinforced Masonry Models. In Proceedings of the 40th International Conference and Exhibition on Computer Graphics and Interactive Techniques (SIGGRAPH 2013). Anaheim.

Pietroni, Nico, Davide Tonelli, Enrico Puppo, Maurizio Froli, Roberto Scopigno, and Paolo Cignoni.. 2015. Statics Aware Grid Shells. Computer Graphics Forum (Special issue of EUROGRAPHICS 2015) 34(2): 627-641.

Rankine, W. J. M. 1862. A Manual of Civil Engineering. London: Griffin, Bohn and Co.

Rippmann, Matthias, Lorenz Lachauer, Philippe Block, P. 2012. Interactive Vault Design. International Journal of Space Structures 27(4): 219-230.

Rippmann, Matthias. 2016. Funicular Shell Design: Geometric approaches to form finding and fabrication of discrete funicular structures. ETH-Zürich.

Rippmann, Matthias. et al. 2016. The Armadillo Vault: Computational design and digital fabrication of a freeform stone shell. In Advances in Architectural Geometry 2016. Zurich: vdf Hochschulverlag.

Schiftner, Alexander and Jonathan Balzer. 2010. Statics-Sensitive Layout of Planar Quadrilateral Meshes. Pp. 221-236 in: Advances in Architectural Geometry. Vienna: Springer.

Schwartz, Thibault and Lucia Mondardini. 2014. Integrated Design and Robotized Prototyping of Abeille's Vaults. Pp. 199-209 in: W. McGee and M. Ponce de Leon, eds., Robotic Fabrication in Architecture, Art and Design 2014. Cham: Springer International Publishing.

Tam, T. K. H. and C. G.Armstrong. 1991. 2D Finite element mesh generation by medial axis subdivision. Advances in Engineering Software 13(5/6): 313-324.

Tessmann, Oliver. 2013. Interlocking Manifold Kinematically Constrained Multi-material Systems. In Advances in Architectural Geometry 2012. Vienna: Springer Vienna, pp. 269-278.

Van Mele, Tom, Andrew Liew, Tomas Mendez, Matthias Rippmann. COMPAS: A framework for computational research in architecture and structures. https://compas-dev.github.io/, 2017. 
Block, P., Lachauer, L. \& Rippmann, M., 2014. Thrust Network Analysis - Design of a cut-stone masonry vault. In S. Adriaenssens et al., eds. Shell Structures for Architecture: Form Finding and Optimization. London

Matthias Rippmann has been a member of the BRG since 2010, where he obtained his doctorate in 2016. Currently, he is a postdoctoral researcher, leading the BRG's digital fabrication research in the NCCR Digital Fabrication. He conducts research in the field of structurally informed design and digital fabrication. He is the developer of the form-finding software RhinoVAULT, which offers TNA-based exploration of funicular shells. He studied architecture at the University of Stuttgart and the University of Melbourne. He worked in Stuttgart at Behnisch Architekten, LAVA, the Institute for Lightweight Structures and Conceptual Design and Werner Sobek Engineers. In 2010, he co-founded the architecture and consultancy firm Rippmann Oesterle Knauss GmbH (ROK).

Philippe Block is professor and co-director of the BRG, and is the director of the NCCR Digital Fabrication. He studied architecture and structural engineering at the Vrije Universiteit Brussel (VUB) in Belgium and at the Massachusetts Institute of Technology (MIT) in the USA, where he earned his PhD under the guidance of Prof. John Ochsendorf in 2009, developing Thrust Network Analysis (TNA), an innovative approach for assessing the safety of historic vaulted structures with complex geometries and designing compression-only shells. With the BRG and as partner of the consultancy Ochsendorf DeJong \& Block (ODB Engineering), he provides structural assessment of historic monuments and design and engineering of novel compression shell structures. 